\title{
Prevention of disease in the tropics: questions in health economics
}

\author{
DAVID J. BRADLEY \\ From the London School of Hygiene and Tropical Medicine
}

No major problem in the health economics of developing countries has been adequately solved; the vast majority of issues have not even been approached. In this paper, therefore, questions are raised and an agenda provided for research; there is no review of the subject. Many existing economic studies are so slight that detailed review would be unhelpful, and my expertise is not in economics.

The main focus of community health in developing countries is now on an exciting and rapidly-some would say too rapidly - changing field. The first part of this paper is a review of what have been considered the key issues and of the types of economic questions raised by the key issue at each stage. The second part is an attempt to consolidate these issues in a systematic manner. This may appear too general a framework, but it has one specific advantage: the economic data base is defective to an extreme degree in most developing countries, and the more dramatic pieces of bad health economics have resulted from a narrow view of the subject in which precise conclusions have been drawn from imaginary data considered in isolation from broader social, cultural, and institutional issues. No doubt such problems also apply to other countries, but in the tropics, where subsistence agriculture is a major occupation and apparent underemployment is frequent, they loom exceptionally large.

THE SCALE OF THE HEALTH PROBLEM

To compare medical problems in developed and developing countries is not easy, because a main feature of underdevelopment is the absence of statistical data. However, some countries do at least have information on deaths in relation to age and the resulting picture is shown in Fig. 1. If we compare survival of 100 babies born in a rich western European country with that of a similar group born in an impoverished African nation, the difference is striking. If deaths show this variation, morbidity and sheer misery are likely to be equally extreme, as well as disability and effects on productivity. A closer comparison of age-specific mortality ratios in the 1960s between Sri Lanka and Sweden underlines the discrepancy and the particularly large differences in the first few years of life (Fig. 2).

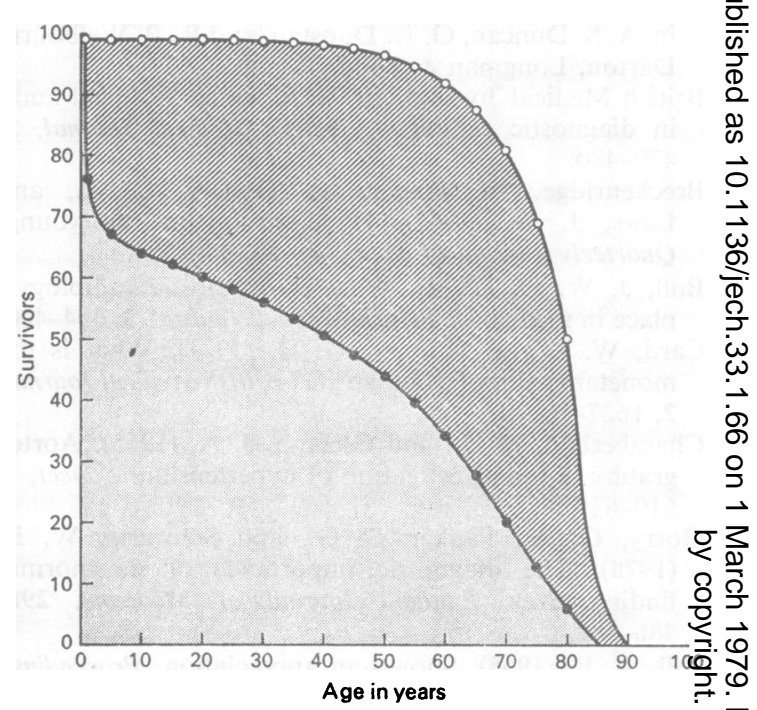

Fig. 1 Survivorship curves for 100 live-born babies in a prosperous industrial country (open circles) and a poor developing country (solid circles).

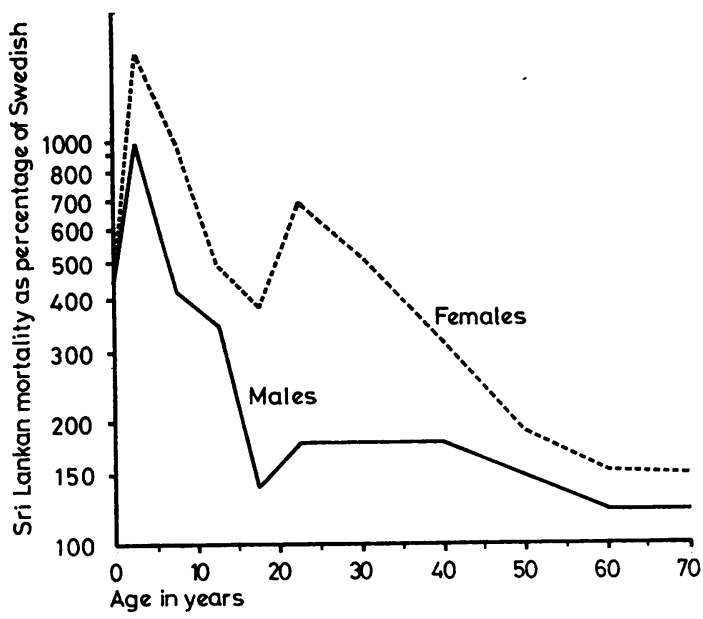

Fig. 2 The ratio of age-specific mortality in Sri Lanka to that of Sweden. (Myrdal, 1968).

Figs. 1 and 2 appear by kind permission of Blackwell Scientific Publications Limited, Oxford. 
If the area between the two survival curves in Fig. 1 represents the tropical health problem, what are its causes? Two predominate: infectious disease and malnutrition. The bulk of tropical problems fall within these two broad categories, though the measures for their control and prevention are varied.

\section{NATURE OF THE HEALTH PROBLEMS}

The countries referred to in this paper are variously known as 'tropical' or 'developing'. Neither term is strictly correct, but each points to one major aspect of the health problems involved. Tropical countries, because of the high ambient temperature, can support many disease agents with a life cycle that involves an obligatory stage or stages of development outside the the human body, either in the physical environment or in the bodies of invertebrate animals. This external development is usually called the 'extrinsic cycle'. For it to occur, warmth is required to keep the invertebrate host from dying, or for development within a reasonable period in the soil. Such diseases include malaria (which kills up to $10 \%$ or more of all babies born in West Africa), other protozoal infections such as sleeping sickness and kala-azar, yellow fever, and the chronic warm climate infections such as schistosomiasis, hookworm, and filariasis. So there are numerous acute and chronic major infectious diseases specific to warm climates.

The diseases of poverty constitute the other great group of health problems in developing countries. A list of the predominant diseases in an English town during the industrial revolution would closely resemble those seen in a tropical outpatient department today. With the exception of malaria, the predominant tropical infections would be severely reduced by raising living standards in a balanced manner. Gastroenteritis, typhoid, cholera, many intestinal worms, infective skin disease, inflammatory eye disease, and dysentery, can all be controlled by improvements in water supply and sanitary excreta disposal rather than by medical action. Similarly, malnutrition can be prevented by food, education, and an adequate income far more effectively than it can be treated, no matter how good the health care facilities.

It has been usual to group together the Third World countries as 'tropical' or 'developing', and certainly the majority of developing countries-or 'less developed countries' as Myrdal (1968) would correctly insist on calling them-are in warm climates. But the position is rapidly approaching where differences between them are more apparent than similarities. The oil-rich Arab countries are clearly a special case, but between the poorest
African states and India, or either of these and southern America, there are profound differences.

\section{THE DEVELOPMENT OF COMMUNITY}

\section{HEALTH ISSUES}

\section{Changing issues in tropical health}

Extensive traditional health care systems have existed in tropical countries for many centuries; these will be referred to below. The early colonial era is a more relevant starting point for the purposes of this paper. Initially, the function of the few medical officers provided was to care for the health of expatriates: traders, the military, and administrators. Aims were clear, the medicine was largely curative, often unsuccessfully so, and the continuous complaint of inadequate funds was the chief economic issue.

The end of the nineteenth century was characterised by the establishment of the germ theory of disease and by a series of massive outbreaks of epidemic disease among the indigenous colonial populations on a scale too great for administrations to ignore. Sleeping sickness killed a third of the people of Uganda, kala-azar killed hundreds of thousands in India, plague and cholera led to devastating epidemics, and famine was a recurrent emergency. Research was both a necessity and, often, a cheap substitute for medical action. Funds were made available on a small scale and interest focused on minimising the budget rather than on formal health economics. As administrations consolidated, the medical officer allocated to each district (of perhaps half a million people) extended his concern from expatriates to the local population, and exiguous curative services at the district hospital were augmented by rural dispensaries and basic public health measures of water supply and sanitation for the headquarters settlement.

The first steps leading to current issues in health economics arose from the successful development of drug treatment for a few of the major tropical disorders, particularly yaws and sleeping sickness in western Africa. Injections of bismuth and then the dramatically effective penicillin for yaws led to the development of itinerant teams of health workers who would travel through endemic areas without any organised health services, examining each village population and giving a single penicillin injection to each person with clinical signs of yaws. Devastating sleeping sickness epidemics were wiping out whole villages in northern Ghana and Nigeria, and these were tackled similarly, though the task was more sophisticated. Here it was necessary to examine people clinically, to puncture enlarged lymph glands, to carry out smears to look for trypanosomes, and to put a needle into the spinal canal to look for 
trypanosomes in the cerebrospinal fluid. This procedure requires a rigorous sterile technique and ideally should be carried out in hospital. Those who were infected needed a course of intravenous injections of toxic medicine. The teams carried out all these procedures in remote villages with notable success. The teams comprised local, often illiterate, people who were given meticulous training in precisely defined procedures with a clear division of labour. The result was a cheap, effective, service to combat a single major disease. When the disease became rarer, visible returns on investment were reduced and the workload fell. Other less dramatic diseases were added to the work of the teams but morale was harder to maintain and the pressure for a more sedentary life increased.

Perhaps the most dramatic campaign against a single disease is just ending. Smallpox eradication was such an effort. Originally the aim was to raise vaccination levels in populations to a level at which herd immunity would end transmission. The later and effective strategy involved case-finding and vaccination of all contacts. Workers were diverted from other health services, the cost was very great, and extreme measures were employed such as rewards for case detection, use of helicopters, and single-minded pursuit of contacts for vaccination. World eradication now appears to have been achieved, the annual savings to countries maintaining port and airport quarantine and inspection facilities are now immense, and few would doubt the benefits of the programme.

\section{Malaria eradication and health economics}

The attempt at world malaria eradication has been even more wide-ranging and expensive. This again derived from a technical advance, the discovery of the long-lasting insecticidal properties of DDT, and the observation that after several years of vigorous malaria control by DDT, if the spraying programme ceased, the malaria might not return (it had died out) although the mosquitoes did. In the 1950s the administrative and technical design of an eradication programme was elaborated. Its nature, with a capital budget for a time-limited programme, perfection of execution necessary in terms of population covered, and staff wholly committed to the malaria programme, has conditioned the main lines of debate in tropical health care for the 1960s and early 1970s. The programme was distinct from other Ministry of Health operations in most countries: it was separate from a very high level downwards, and its budget was autonomous. The programme had a variable success: there is now no indigenous malaria in Europe and the United States of America and it has been eliminated from many other countries. On the other hand, eradication never got under way in Africa for good biological reasons. More important in the present context, it was relatively successful in India, Sri Lanka, and other parts of south-east Asia, but then collapsed, partly because insecticide resistance developed among the vector mosquitoes, but mainly because the health service infrastructure was unable to support the immense task of regular surveys of the possibly infected people in the later stages of the campaign. A vertically structured capital programme collapsed as the result of general deficiencies in the health care sector.

This historical account has been given because it explains much of the present situation in health economics of the tropics. Before and during the malaria eradication programme, tropical health administrators rarely thought consciously in terms of economics. There was a health budget to be eked out. It was so minute in size compared with the health needs of the population that the feel for what was practicable dominated expenditure, and maintaining services during the slump period was a matter of daily exigencies rather than economic planning. It was different with malaria eradication. The sums required were vast. They were certainly not available from health budgetary savings, and they were capital not the recurrent sums needed for ordinary controte The programme relied on international expertise and depended on the World Health Organisation (WHO for leadership. The proponents of malaria eradication found themselves having to convince the Treasury, not the Health Ministry, because extrabudgetary funds were needed. So economic rather than humanitarian arguments were needed, and the WHO had the strongest of motives for costing malaria as highly as possible. Thus a pattern of economic justification was set for single disease programmes which has taken such deep roots in that organisation, and hence in health economics in developing countries, that even today it is difficult for many in the WHO to perceive more than this crude form of cost and benefit calculation when the words 'socioeconomic aspects' are used!

The costing of disease, especially malaria, in the tropics has much earlier beginnings in the hands of Ross and especially Watson at the beginning of the century. Mosquitoes had been shown to carry malaria; drainage could keep down mosquitoes. Malaria was exacting an immense toll in absenteeism, disability and death. Commercial interests in Port Swettenham were at stake. Watson was talking to planters, mine owners, and merchants about their labour forces. The Ross Institute, which he subsequently directed, continued to do so. A straightforward calculation was needed to show that to include malaria control in the balance sheet would 
raise profits. This was performed, appropriate action was taken, and profits rose. The actions requireddrainage and oiling of swamps-were engineering jobs and were technically relatively self-contained. Malaria was so predominantly the hazard that many of the sophistications of health economics could be by-passed. Such arguments also prevailed on the Zambian copper belt in the 1930s. At that time, also, Sinton made his calculations on the cost of malaria to India, while examples accumulated of good land made uninhabitable because of malaria and of dramatic rises in land prices following malaria control.

There was thus a tradition upon which the economic case for malaria eradication could be erected. Furthermore, in those places where the programmes were planned, malaria was an acute fever producing disability comparable in some ways to an influenza epidemic, and a clear mortality. The calculations were made and the funds voted. With the exception of smallpox, the economic issues are unlikely again to appear so simple. Other diseases raise more complex problems and malaria also is now seen to be less straightforward in the long term. It may be helpful to illustrate some aspects of the problems raised by single disease questions before examining the broader issues of health care and combinations of the two.

\section{Costs of diseases and their control}

In many endemic areas malaria kills small children on a large scale. Therefore once eradication was in sight, analysis of the demographic consequences was undertaken, particularly in Sri Lanka, where only 16 people got malaria in 1963. Large consequences were found, with adverse long-term economic results following from the increase in population.

The second disease for which energetic attempts at costing were made illustrates practically all the difficulties that malaria work contrived to avoid. Schistosomiasis is a disease caused by a helminth, which is a sort of worm. Many of those infected have mild symptoms, or symptoms which, though distressing, do not prevent the person from working, or no symptoms at all. A proportion, difficult to measure, go on to cause permanent damage to various body tissues and as a result some people die of the disease. Evidence on the working capacity of those apparently without symptoms is conflicting. Several parasite strains exist and possibly people vary in their susceptibility to late effects. People have differing worm burdens which depend on exposure to infection and on immunity, which is ill-defined in man, and these worm burdens greatly affect the disease risk. The economist therefore has extremely complex, uncertain material as a starting point.
He also has to face the difficulty of costing misery, and partial disability in a subsistence economy.

In both malaria and schistosomiasis the initial impetus for study tended to be advocacy: 'Go and show how very important this disease is'. With so many uncertainties in any calculation, the initial cost estimates have been high, followed by an iconoclastic series of publications arguing the contrary. Also, more sophisticated studies have been directed towards areas of lower infection intensity for schistosomiasis.

Recently, similar calculations have been made for Ascaris, roundworm infections in Kenya. These have served a useful purpose in drawing attention to an underrated disease problem; but as with the studies of schistosomiasis, there is the difficulty of estimating the large and uncertain cost of the consequences of subclinical and non-specific pathological effects.

With the decreasing enthusiasm for 'vertical' single disease campaigns, there may be less pressure for the preceding types of cost/benefit estimates; the more so, as economists and planners absorb the physicians' scepticism about such data. A more promising subject for economic analysis is onchocerciasis, because blindness is an unequivocal disability and the effects of river blindness on agriculture and even on the habitability of some areas may be influenced. The multinational West African programme of onchocerciasis control is funded by the World Bank (IBRD).

The Development Banks might be expected to be the chief proponents of cost/benefit calculations on disease, but this has not been so. The IBRD, although much concerned about health, does not, at the time of writing, lend money for health projects as such. Its interest is confined to population projects with a bearing on health or development of water resources etc., when such development would affect health. The purpose of the Bank's very large funding of schistosomiasis control has been to prevent ill health resulting from water development, and so to reduce the impact on health of environmental change; it has not been its purpose to provide medical care, so it has not required detailed benefit calculations

\section{Organisation, quality, and coverage of health care services}

Malaria eradication at global level failed for many reasons-biological, technical, and administrative. In those countries of South-east Asia, where it almost succeeded but then collapsed, a major cause was the lack of a general health infrastructure of skilled or semi-skilled workers capable of carrying out the demanding task of following up the whole population in the late stages of the campaign when few cases 
of malaria remained. Subsequently the WHO transferred its attention to building up the basic health services needed for most attacks on particular diseases. The emphasis was on management and on provision of health centres along with training of health staff. The period coincided with the growth of local medical schools in tropical Africa and an attempt to augment the exceedingly low doctorpatient ratios.

It quickly became clear that there were too few doctors, that to educate doctors was expensive, and that even if enough doctors were trained, there would be inadequate funds to employ them in rural areas, where they had no wish to live.

Preoccupation with the problem of how to cover the population with some form of basic health care system has steadily increased up to the present time. At first the aim was to provide health centres in which preventive and curative medicine could be practised by a health team led by the doctor. The costs of manpower training and employment for different categories of staff were compared and the immense cost of doctors became apparent.

More recently, two developments of the 'coverage' approach have been emphasised. The first is 'country health planning', in which a plan is prepared for development of health services at national level. Such a plan is amenable to economic analysis and rational discussion, even if these do not greatly influence the decisions subsequently taken. The second development is an emphasis on village community health workers (CHWs) and self-help programmes. The $\mathrm{CHW}$ is often thought of as someone with little training who may do unpaid or part-time work. Nevertheless the CHW is expected to do a great deal with very limited support. Programmes based on CHWs have had considerable success on a small scale, and in particular political contexts on a larger scale. Their promotion is being strongly urged by the WHO but their use raises several points relevant to this paper.

The value of $\mathrm{CHWs}$ at national and village levels has been studied, but their value at intermediate levels, and especially that of the district, has been neglected. Programmes which have not been institutionalised at district level will not continue or be replicable. A variety of economic questions arise: how does one cost a community health worker and assess the benefits of his or her work?; are the numerous calculations valid that show a very high cost-effectiveness of community-based piogrammes compared with hospital programmes? Among other questions which have scarcely been studied are the optimum allocation of funds between the tiers of the health hierarchy; the issues surrounding remuneration of CHWs; and the allocation of funds as between staff, preventive activities, and medicines. At every level of the health service, measures of the output of health improvements are neglected, and most economic work has to be based on measuring the utilisation of facilities. One exception is the work of the Ghana Health Ministry planning unit, as yet unpublished, which is discussed below.

\section{Environmental health costs}

Analyses of improvements in water supply and the disposal of excreta lie somewhere between single disease studies and the economics of the whole health sector, although extending beyond the health field itself. Recently several efforts have been made to estimate the economic benefits of improvements in health resulting from improvements in water supply, especially partial ones. The problems have been great. In particular, the medical data on the relation of infection to disease, and on the likely benefits of improvements when assessed in medical terms, are inadequate as a basis for serious economic analysis. More successful attempts have been made to compare public health engineering changes with immunisation and treatment for particular waterrelated diseases, notably typhoid.

\section{Health and development}

It has been increasingly realised that health cannot be considered in isolation from other problems irf developing countries, and this has led to integrated rural and urban development projects often funded now by international banks such as IBRD. Disaggregation of the health component for economic analysis is difficult, without heroic assumptions.

The converse problem, of deteriorating health as a result of economic development, is widespread. It is most clearly seen in water development projects, especially man-made lakes and irrigation schemes. Health costs need to be added to the other costs of development, although in practice the matter has been handled-if at all-by setting aside an arbitrary sum for mitigating undesirable effects of the development. In addition, design modifications have been made to reduce disease transmission, provided the cost was low. Thus the economics of health as a component of development have not been handled in a systematic manner, partly for the good reason that too many medical as well as economic uncertainties are involved.

THE BASIC QUESTIONS

Data available and needed

In the preceding section I have outlined the history of the major preoccupations of community health and hinted at the economic issues which have been raised but remain unanswered. If the subject is to 
progress, more co-operative work will be needed between the economist and the epidemiologist. The tendency has been for policy to be based on utilisation of facilities and not on measured health improvements. In the present climate of opinion, when issues of coverage overshadow those of quality of care, it is clearly essential for economics to be based on the actual changes in health status if understanding is to be improved.

If resources are minute, and the total health budget of several developing countries is below $£ 1$ per head per annum, complete solutions to many problems of disease control are not feasible. Therefore economic choices are harder, and the need for epidemiological understanding much gieater, than in developed countries: if there are funds only to improve either access to water or its quality, then the demand for epidemiological knowledge is very great indeed. How much will diarrhoea decrease if standpipes replace the local pond as a water source? What further reductions will there be as a result of doubling the number of standpipes, compared with moving to single household taps?

\section{Methods for determining costs of diseases and their control}

The malariologists' approach to disease economics -namely, adding up the total social cost of a disease and treating that sum as the benefit from eradication-is relevant only to eradication, if then. Where a disease will be controlled or its frequency somewhat reduced, a different approach is needed. If we consider a complex helminthic disease such as schistosomiasis, the risks of infection, of transition to a series of disease states, and of death, may all be set out, and disability and economic consequences estimated for each. A second method attempts to bypass the medical issues and to compare economic productivity in those infected with a control group which is uninfected. Where such productivity is measurable, bias is introduced, because the workforce is usually a selected group and may well exclude all those seriously affected by the disease under study, as has been shown with onchocerciasis in Uganda. To obtain appropriate control groups for comparative purposes is also difficult. Where a disease is curable, the best approach is likely to be a comparison of two groups, with and without the disease, both before and after the affected group has been treated. Economic extrapolation from such studies to the whole community requires some heroism, but where subsistence agriculture and apparent unemployment dominate the rural scene, problems of design are not readily solved.

Working out the costs of actual control procedures is a great deal easier, although it is not often done. It raises problems familiar to the economist for which acceptable approaches exist. The cost of treatment can also be worked out. The major difficulty with most endemic diseases is that the straightforward costs of this type are small, relative to the intractable level of disability which forms the largest component of total social cost; I would almost assert that the standard deviation of the estimates of disability often exceeds the total of other costs, so that what is reliably measurable in many diseases is relatively trivial.

The many methods devised in developed countries for estimating the cost of loss of life and disease have been sporadically applied to tropical diseases. Some achieved notoriety, as when the enormous cost of diarrhoeal disease in one calculation was shown to be due to loss of earnings by the sick, calculated as days $\times$ GNP per head per day despite the fact that most of the sick were under three years old. In a recent attempt in Ghana, the mean days of life lost from each disease by each age group compared with the mean expectation of life of that age group in Ghana have been calculated. This approach is of course vulnerable to criticism, but it has been used in health planning, and except for a tendency to over-value life in the newborn, it appears to be really useful.

\section{Health economics for planning}

In the Ghana planning unit, Nimo, Morrow, Smith, and others have used the relative importance of different diseases, calculated as described above, in assessing priorities. The unit has also estimated the changes in lost-days-of-life from various intervention strategies and thereby assessed the costeffectiveness of several approaches to health care improvement. The results strongly support a primary health care strategy as against the alternatives. Although the authors of this planning method would be the first to admit its limitations, it appears to represent a considerable advance on the way in which plans have been developed in other tropical countries.

It is clear that in developing countries, where lack of funds severely limits the range of health care activities, as informed an analysis as possible is needed as a basis for planning. The same questions are being asked as in the United Kingdom, but they need to be tackled in a very different way. It is hoped that continuing discussion will clarify the most helpful lines of progress.

\section{Comment: NICHOLAS M. PRESCOTT Magdalen College, Oxford}

My comments will be addressed primarily to a fundamental question which Bradley touches on but does not 
confront directly: what is the appropriate approach to health sector resource allocation in developing countries (LDCs)?

Bradley correctly suggests that the application of economic analysis to health in LDCs has been limited and sometimes of questionable value. What he did not say was that few of these applications have been made by economists. This is not a trivial observation. The enormity of the health problems in LDCs and the extreme scarcity of resources available to deal with them give an importance to efficient resource allocation which is not well served by much of the LDC health economics practised in the past. How can economics improve the efficiency of resource allocation in the future? Few would disagree in general terms with the answer contained in Bradley's concluding comment that '.. as informed an analysis as possible is needed as a basis for planning'. But it begs the questions both of the type of economic analysis that is needed for health planning and of the nature of the relevant information.

One possible planning approach follows from the currently fashionable 'basic needs' approach to development (Ghai et al., 1977), the latest product of the shift in emphasis from growth to distribution as the objective of development planning. This approach is distinguished by its emphasis on meeting the basic needs of the poor in the shortest possible time, say 15 to 20 years. Health is one of the five commonly identified elements of the core bundle of basic needs; the others are food, education, housing, and water supply and sanitation. A natural interpretation of the basic needs strategy is that it implies a cost-effectiveness approach to health planning: targets for basic health needs are specified somehow and the planning problem is reduced to identifying the least-cost techniques for meeting them. There are evident difficulties with this approach. For example, what are the basic needs in health? Should they be formulated in terms of resource inputs or of health status outputs? Who decides and on what basis? Abstracting from these difficulties, it is natural to ask what might be the cost of a 'reasonable' basic needs stategy in health? To illustrate the possible orders of magnitude the World Bank has attempted to estimate the cost of an input plus quasi-output strategy for meeting basic health needs by the year 2000: extension of primary health services to the urban and rural poor, and eradication of four of the major tropical parasitic diseasesmalaria, schistosomiasis, onchocerciasis, and African human trypanosomiasis (Burki and Voorhoeve, 1977). The results indicate that the marginal costs of meeting basic health needs so defined would lie in the region of US $\$ 2$ to $\$ 3$ at 1975 prices per caput per year over the period 1980 to 2000 , perhaps a doubling of government health expenditure per caput in many LDCs. The crudity of this figure should not be underestimated but it does suggest that meeting basic health needs will require reallocation of domestic and international resources on a scale that is unlikely to be achieved in the near future. The implication is that choices have to be made even between basic health needs in many LDCs-choices which are bypassed by the basic needs approach. These choices can be resolved rationally only by explicit and systematic comparison of the benefits and costs, the net contribution to social welfare, of alternative health programmes.

Yet the use of cost-benefit analysis to improve the efficiency of resource allocation is hindered by misunderstanding of the nature of the information that is relevant for planning. Two observations will suggest areas where substantial progress is required.

The first observation is that economic analysis has been widely misinterpreted to be concerned only with the aggregate income benefits that may result from health status improvement while ignoring the social value of the aggregate health benefits themselves. This misinterpretation has led to the perverse distinction often drawn between the 'economic' and the 'humanitarian' benefits of disease control; it is also reflected in the term 'socioeconomic development'. In so far as health status improvement is itself an ultimate objective of development planning, the question is not whether but how aggregate health benefits should be integrated with aggregate income benefits in the appraisal of health programmes. This raises the problem both of quantifying, and of valuing relative to a common numeraire, the different types of aggregate health benefit. Much remains to be done to develop the pioneering attempt by Feldstein et al. (1973) to aggregate income and health benefits in a single objective function for health sector planning in LDCs. The attempt by the Ghana planning unit to quantify the aggregate health benefits of alternative planning strategies appears to be a valuable step in this direction. In addition it will often be appropriate to attach a distributional premium to income and health benefits accruing to beneficiaries in the lower income groups.

The second observation is that past attempts to evaluate the benefits of disease control by estimating the 'economic loss' due to disease have tended to assume implicitly that disease would be completely and instantaneously eradicated. This assumption ignores the choice that exists in the technology of disease control. The expected benefits of control depend fundamentally on the expected changes in mortality and morbidity, and their distribution by age, sex, and income class, in future periods. These factors vary with the choice of control technique and the scale at which it is operated. Yet the possibility of identifying the optimal choice of technique and scale of control (the objective of which may fall far short of eradication) is at present precluded by the lack of a solid epidemiological basis for predicting the dynamic response of disease prevalence to different kinds of intervention; this problem is well illustrated by Bradley's example of choice of technique in water supply. Among the major tropical parasitic diseases, only schistosomiasis has been the subject of epidemiological modelling in a planning framework-but one of costeffectiveness analysis (Rosenfield et al., 1977).

In summary, the cost-effectiveness approach exemplified by the basic needs strategy cannot resolve health sector choices in the face of the severe scarcity of resources in many LDCs. Furthermore, cost-benefit analysis cannot significantly improve allocative efficiency until future research has established the basis for predicting, quantifying, and valuing the expected aggregate health 
benefits of alternative health programmes. These are substantial requirements which call for close collaboration between economists and epidemiologists. But they are essential for rational planning of the health sector in pursuit of the objectives of economic development.

Reprints from Professor David J. Bradley, Director of the Ross Institute of Tropical Hygiene and Professor of Tropical Medicine, London School of Hygiene and Tropical Medicine, Keppel Street, London WC1E 7HT.

\section{References}

Burki, S. J., and Voorhoeve, J, J. C. (1977). Global estimates for meeting basic needs: background paper.
Basic Needs Papers No. 1 (mimeo). World Bank: Washington DC.

Feldstein, M. S., Piot, M. A., and Sundaresan, T. K. (1973). Resource allocation model for public health planning. A case study of tuberculosis control. Bulletin of the World Health Organisation, 48, (Supplement).

Ghai, D. P., Khan, A. R., Lee, E. L. H., and Alfthan, T. (1977). The Basic Needs Approach to Development. International Labour Office: Geneva.

Myrdal, G. (1968). Asian Drama: An Enquiry into the Poverty of Nations, vol. 2, p. 1412. Twentieth Century Fund: New York (copyright) and Penguin: London.

Rosenfield, P. L., Smith, R. A., and Wolman, M. G. (1977). Development and verification of a schistosomiasis transmission model. American Journal of Tropical Medicine and Hygiene, 26, 505-516. 